\title{
Low cerebrospinal fluid insulin levels in obese humans
}

\author{
W. Kern • C. Benedict • B. Schultes $\cdot$ F. Plohr • \\ A. Moser • J. Born • H. L. Fehm • M. Hallschmid
}

Received: 21 July 2006 / Accepted: 25 July 2006 / Published online: 2 September 2006

(C) Springer-Verlag 2006

\section{To the Editor:}

In healthy men, insulin concentrations in cerebrospinal fluid (CSF) increase after acute elevations of plasma insulin levels, indicating that the brain content of insulin is normally finely tuned to circulating insulin [1]. Central nervous insulin promotes weight loss [2] and attenuates peripheral insulin resistance via inhibition of hepatic gluconeogenesis [3]. With increasing body weight, plasma insulin levels rise as a result of elevated insulin resistance. It is not yet known whether in hyperinsulinaemic obese subjects this increase is accompanied by parallel increases in CSF insulin levels that would act to promote weight loss in these patients.

We compared insulin concentrations in plasma and CSF in a group of 45 subjects ( 23 women, age $19-80$ years) with a wide body weight range (BMI $16.24-38.10 \mathrm{~kg} / \mathrm{m}^{2}$ ). Five subjects were newly diagnosed as diabetic by elevated fasting plasma glucose levels $(>7.0 \mathrm{mmol} / \mathrm{l})$. Since their exclusion did not alter the results, their data were included

W. Kern • B. Schultes $\cdot$ F. Plohr $\cdot$ H. L. Fehm

Department of Internal Medicine I, University of Luebeck, Luebeck, Germany

C. Benedict $\cdot$ J. Born $\cdot$ M. Hallschmid $(\square)$

Department of Neuroendocrinology, University of Luebeck, Ratzeburger Allee 160,

23538 Luebeck, Germany

e-mail: hallschmid@kfg.uni-luebeck.de

\author{
A. Moser \\ Department of Neurology, University of Luebeck, \\ Luebeck, Germany \\ B. Schultes \\ Obesity Center, Spitalregion St Gallen-Rorschach, \\ St Gallen, Switzerland
}

in the analyses. After an overnight fast, subjects reported to the laboratory for simultaneous sampling of blood and CSF $(1 \mathrm{ml})$ via lumbar puncture after local anaesthesia $(2 \mathrm{ml}$ mepivacain- $\mathrm{HCl} 1 \%$ ). BMI and waist-to-hip ratio were assessed, and body composition was measured by standard bioelectrical impedance analysis (BIA 2000-M; Data Input, Frankfurt, Germany). Insulin was determined using a commercial competitive double-antibody RIA (Pharmacia Insulin RIA 100; Pharmacia Diagnostics, Upsalla, Sweden). To increase the sensitivity of the insulin assay for the expected lower concentrations in CSF, the standard procedure was slightly modified. In brief, insulin was determined using $100 \mu \mathrm{l}$ of CSF, $50 \mu \mathrm{l}$ of $\left[{ }^{125} \mathrm{I}\right]$ insulin diluted with buffer at a ratio of $1: 3$, and $50 \mu \mathrm{l}$ of insulin antiserum diluted at a ratio of 1:2. The incubation time was increased from $2 \mathrm{~h}$ in the standard method to $3 \mathrm{~h}$ at room temperature. With this procedure, threshold sensitivity was reliably lowered to $1.8 \mathrm{pmol} / 1$. Intra-assay variation was $<4.5 \%$ for determination in CSF and plasma. Insulin plasma and CSF concentrations were measured in duplicate and quadruplicate, respectively. The lowest standard used was $1.8 \mathrm{pmol} / \mathrm{l}$, which could be reliably measured with $<4.5 \%$ variation. Therefore, all measures below this standard were set to $1.8 \mathrm{pmol} / \mathrm{l}$ for analysis. Thus, the actual CSF:plasma insulin ratio may even be slightly lower than reported, although analyses using the measurements below the lowest standard did not alter the results. Additionally, plasma and CSF glucose concentrations were measured (Beckman glucose analyser II; Beckman Instruments, Fullerton, $\mathrm{CA}$; coefficient of variation $<1.1 \%$ ). Insulin resistance was estimated according to the homeostasis model assessment (HOMA) formula, with high scores denoting a high degree of insulin resistance. Bivariate correlation coefficients were determined and a stepwise multiple regression analysis was performed to detect associations 
between the CSF:plasma insulin ratio and the other variables (age, fat mass, sex, BMI, CSF:plasma glucose ratio, waist circumference, hip circumference and waist-to-hip ratio). HOMA scores were log-transformed prior to analysis to achieve normal distribution.

As expected, plasma insulin levels increased with rising BMI $(R=0.511, p<0.001)$, body fat mass $(R=0.471, p<0.001$; Fig. 1a), waist circumference $(R=0.454, p=0.002)$, hip circumference $(R=0.602, p<0.001)$ and degree of insulin resistance $(R=0.913, p<0.001)$. However, the CSF:plasma insulin ratio was negatively associated with $\mathrm{BMI}(R=-0.315$, $p=0.04)$, fat mass $(R=-0.393, p=0.008$; Fig. $1 b)$, waist circumference $(R=-0.315, p=0.04)$ and hip circumference $(R=-0.374, p=0.01)$ and with the degree of insulin resistance $(R=-0.633, p<0.001)$. In the stepwise multiple linear regression analysis with the CSF:plasma insulin ratio as the dependent variable, the degree of insulin resistance was the only factor independently correlated with the CSF:plasma insulin ratio $\left(n=45, R^{2}=0.40, \beta=-0.632\right.$, $\mathrm{SE}=0.011, p<0.001)$, with the degree of insulin resistance explaining $40 \%$ of the variation in the CSF:plasma insulin ratio. In contrast, none of the other variables was independently related to the CSF:plasma insulin ratio $(p>0.123)$.

Our finding of a decreasing CSF:plasma insulin ratio with increasing body weight indicates that obesity in humans, as observed in animals [4], is characterised by a relative central nervous insulin deficit. It may be speculated that the entry rate of circulating insulin into the brain is reduced in obesity, despite plasma insulin levels that are usually distinctly below the level of receptor saturation at the blood-brain barrier [4]. Reduced sensitivity of the insulin receptor at the blood-brain barrier could restrict the entry of insulin into the brain/CSF compartment. This hypothesis is supported by findings that endothelial cells of brain microvessels in obese $f a / f a$ rats exhibit reduced insulin binding [5]. Furthermore, dexamethasone, a synthetic glucocorticoid that induces insulin resistance and weight gain, is known to reduce central nervous system insulin uptake [6]. Fittingly, in our study, insulin resistance was the only parameter independently associated with a decreased CSF:plasma insulin ratio, although high HOMA scores resulting from increased plasma insulin levels may have added to this effect. However, we did not measure insulin binding in blood cells which might have shed more light on the assumption of reduced blood-to-brain insulin transport in obesity.

Whether the reduced CSF:plasma insulin ratio precedes obesity and insulin resistance or vice versa is unclear. Hypothalamic insulin signalling is essential for inhibiting peripheral glucose production [3], and decreased numbers of hypothalamic insulin receptors cause hyperphagia and insulin resistance in rats [2]. Reduced insulin levels in the brain may favour weight gain and increase peripheral insulin resistance, which, in turn, might further hamper brain uptake of insulin and eventually lead to a vicious circle. Insulin and leptin jointly serve as adiposity signals to the brain, and studies on plasma and CSF leptin in obesity have yielded results comparable to our insulin data [7]. On the background of findings that brain insulin also exerts a regulatory influence on neurodegenerative processes [8], future studies should address the question of whether decreased central nervous insulin levels predispose to the development of disorders like Alzheimer's disease and obesity.

Acknowledgement Supported by the Deutsche Forschungsgemeinschaft (German Research Foundation), grant no. SFB-654/B3.

\section{a}

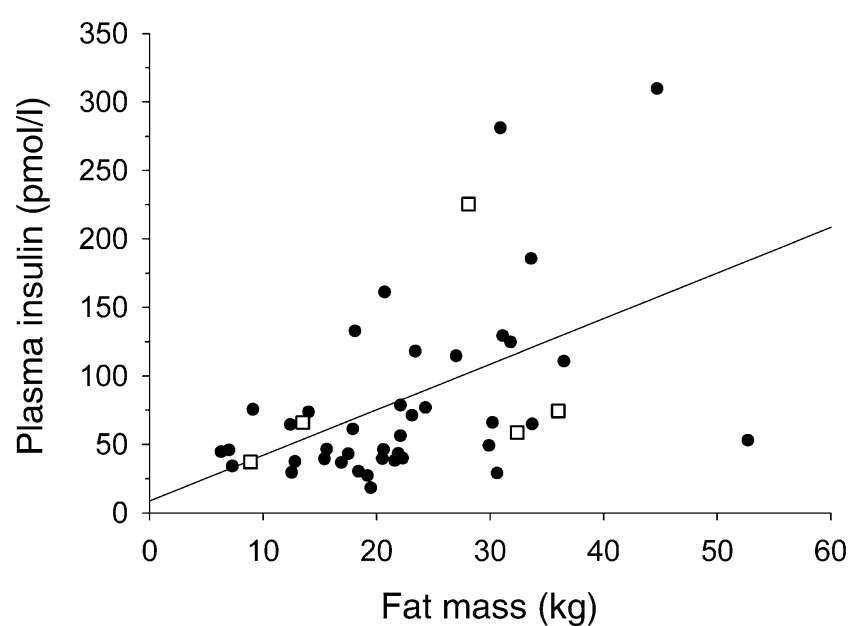

b

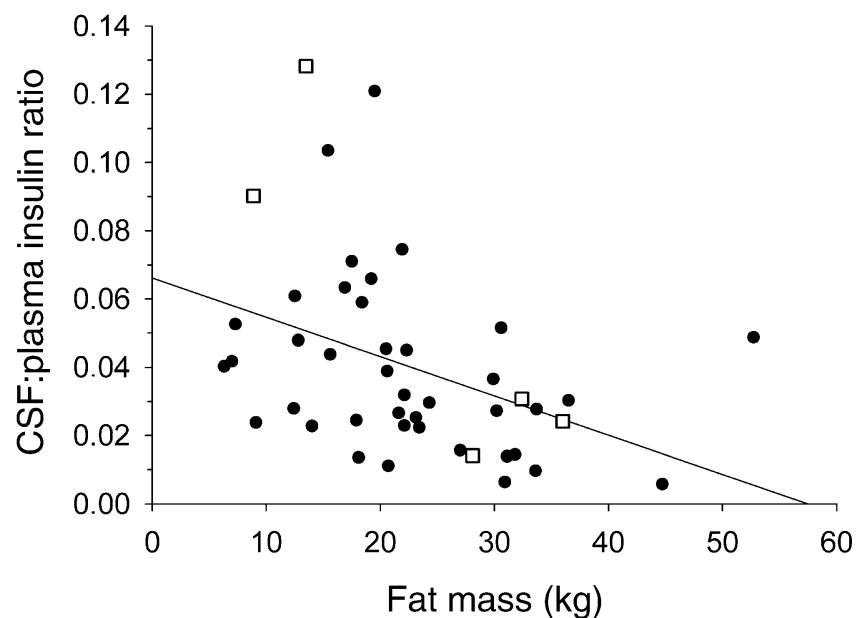

Fig. 1 Correlation of body fat mass with fasting plasma insulin concentration ( $n=45, R=0.471, p=0.001)$ (a) and CSF:plasma insulin ratio ( $n=45$, $R=-0.393, p=0.008$ ) (b). Open squares indicate five subjects newly diagnosed with type 2 diabetes 


\section{References}

1. Wallum BJ, Porte D Jr, Figlewicz DP, Jacobson L, Dorsa D (1987) Cerebrospinal fluid insulin levels increase during intravenous insulin infusion in man. J Clin Endocrinol Metab 64:190-194

2. Obici S, Feng Z, Karkanias G, Baskin DG, Rossetti L (2002) Decreasing hypothalamic insulin receptors causes hyperphagia and insulin resistance in rats. Nat Neurosci 5:566-572

3. Obici S, Zhang BB, Karkanias G, Rossetti L (2002) Hypothalamic insulin signaling is required for inhibition of glucose production. Nat Med 8:1376-1382

4. Woods SC, Seeley RJ, Baskin DG, Schwartz MW (2003) Insulin and the blood-brain barrier. Curr Pharm Res 9:795-800
5. Schwartz MW, Figlewicz DP, Kahn SE, Baskin DG, Greenwood MR, Porte D Jr (1990) Insulin binding to brain capillaries is reduced in genetically obese, hyperinsulinemic Zucker rats. Peptides 11:467-472

6. Baura GD, Foster DM, Kaiyala K, Porte D Jr, Kahn SE, Schwartz MW (1996) Insulin transport from plasma into the central nervous system is inhibited by dexamethasone in dogs. Diabetes 45:86-90

7. Caro JF, Kolaczynski JW, Nyce MR et al (1996) Decreased cerebrospinal-fluid/serum leptin ratio in obesity: a possible mechanism for leptin resistence. Lancet 348:159-161

8. Schubert M, Gautam D, Surjo D et al (2004) Role for neuronal insulin resistance in neurodegenerative diseases. Proc Natl Acad Sci USA 101:3100-3105 